\title{
Is there a familial predisposition to bisphosphonate-induced atypical femoral fractures?
}

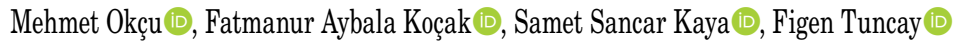 \\ Department of Physical Medicine and Rehabilitation, Ahi Evran University School of Medicine, Kırşehir, Turkey \\ Received: September 12, 2019 Accepted: January 29, 2020 Published online: September 01, 2021
}

\begin{abstract}
Bisphosphonates are commonly used in the treatment of osteoporosis. Atypical femoral fracture (AFF) is a well-known adverse effect of bisphosphonate use. The importance of genetic factors has been demonstrated in bone quality, bone turnover, and in the response to osteoporosis treatment. Herein, we present two cases of bilateral AFFs after bisphosphonate use for a short period of time in members of the same family (mother and her daughter) and discuss genetic predisposition to bisphosphonate-induced AFFs in the light of literature data.
\end{abstract}

Keywords: Atypical femoral fracture, bisphosphonate, genetic factors.

Osteoporosis is characterized by a decrease in bone mineral density and deterioration in microarchitecture. ${ }^{[1]}$ Calcium, vitamin $\mathrm{D}$, bisphosphonates, parathyroid hormone, and denosumab are commonly used in its treatment. Bisphosphonates have been shown to reduce bone turnover, increase bone mineral density, and reduce fracture risk. ${ }^{[2]}$ Although they are usually welltolerated and safe drugs, there are also reports that long-term use of bisphosphonates may lead to atypical stress fractures. ${ }^{[3]}$

In recent years, the importance of genetic factors in the heterogeneity of bone quality, bone turnover, and response to osteoporosis treatment has been examined in many studies. In addition, the risk for exposure to adverse effects due to bisphosphonate use has been shown to have a polygenic basis. ${ }^{[4-6]}$ In this report, we present two cases (mother and daughter) of the same family who developed bilateral multiple atypical femoral fractures (AFFs) after three and four years of bisphosphonate use, respectively.

\section{CASE REPORT}

Case 1- A 60-year-old female patient presented to our outpatient clinic with bilateral thigh pain for two years. She had no history of trauma, weight loss, or fever. She was using acetylsalicylic acid $100 \mathrm{mg}$ tablet, candesartan cilexetil/hydrochlorothiazide $16 \mathrm{mg} / 12.5 \mathrm{mg}$ tablet, metoprolol succinate $100 \mathrm{mg}$ tablet, and trimetazidine dihydrochloride $35 \mathrm{mg}$ for hypertension and coronary artery disease. The patient was started on alendronate sodium $70 \mathrm{mg}$ weekly after being diagnosed with osteoporosis about four years ago and discontinued the drug one year ago. She used bisphosphonate for a total of three years. Her medical history revealed no history of glucocorticoid use. According to her family history, her father had coronary artery disease and hypertension, while

Corresponding author: Mehmet Okçu, MD. Kırşehir Ahi Evran Üniversitesi Eğitim Araştırma Hastanesi Fiziksel Tıp ve Rehabilitasyon Anabilim Dalı, 40200 Kırşehir, Türkiye. e-mail: mehmet.okcu@ahievran.edu.tr 
her mother had a history of anxiety disorder and fractures of both thighs. Physical examination revealed bilateral tenderness in the middle of the thigh without swelling. X-ray images of the patient one year ago and currently showed cortical thickening and transverse fracture line in both femoral middle shafts laterally. These findings were consistent with bisphosphonate-induced AFF, and three of these findings were found in the left femur and one in the right femur (Figure 1). Serum parathyroid hormone $(\mathrm{PTH})$, calcium (Ca), phosphorus (P), alkaline phosphatase (ALP), 25-hydroxyvitamin D [25(OH)2D], hemoglobin $(\mathrm{Hb})$, complete blood count (CBC), erythrocyte sedimentation rate (ESR), and C-reactive protein (CRP) values were found to be normal. Bone mineral density was measured using dual-energy X-ray absorptiometry. The T-score was $-2.5\left(0.778 \mathrm{~g} / \mathrm{cm}^{2}\right)$ in the lumbar vertebra, $-1.5\left(0.850 \mathrm{~g} / \mathrm{cm}^{2}\right)$ in the total hip, and $-1.2\left(0.790 \mathrm{~g} / \mathrm{cm}^{2}\right)$ in the left femoral neck. Medical treatment and a physical therapy program were arranged. The patient was informed that she should not take bisphosphonate and about possible risk for falling. The orthopedics department was consulted for surgery, and the patient was scheduled for an operation. However, she refused surgery. She was discharged with recommendations. No deterioration in her fracture was detected at subsequent visits.

Case 2- An 83-year-old woman, the mother of Case 1 , had a history of atypical fractures in both femoral

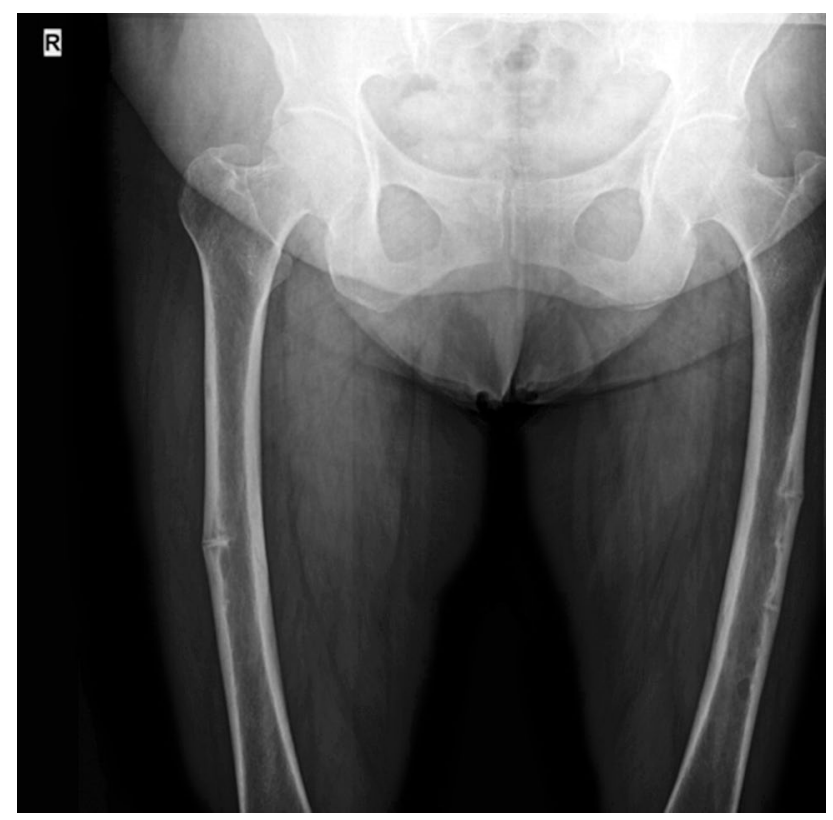

Figure 1. Bilateral atypical femoral fractures of Case 1. mid-shaft and right subtrochanteric regions at four different times without any trauma after four years of bisphosphonate use. She had occasional mild hip and

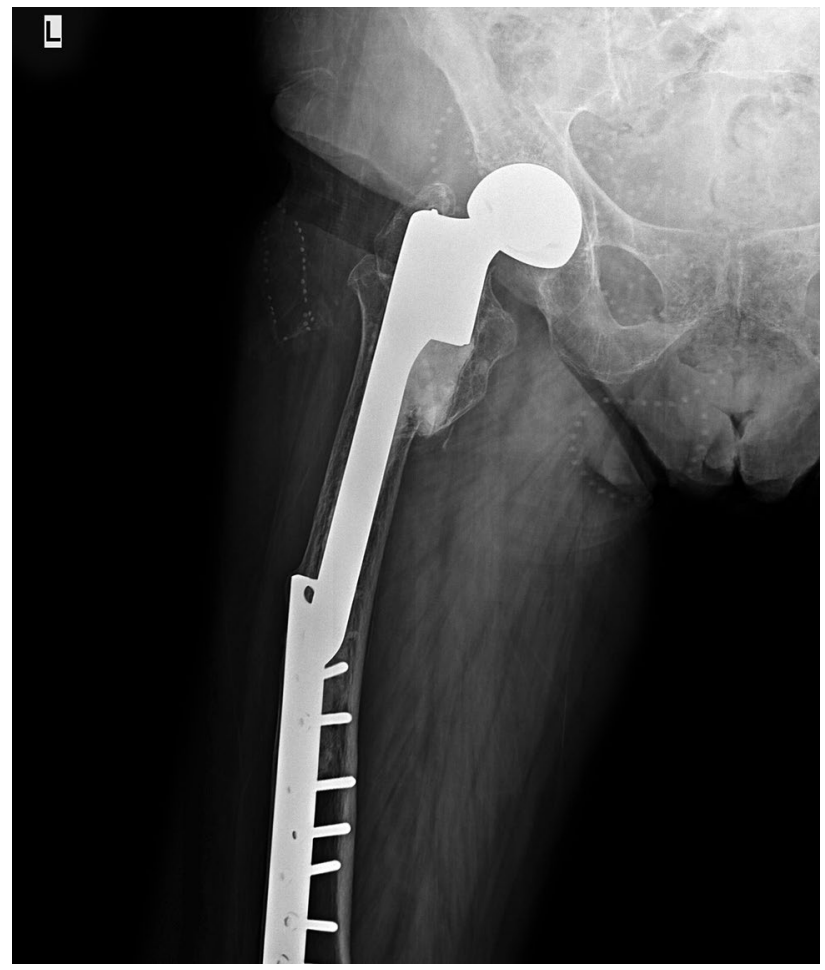

Figure 2. X-ray image of Case 2 (right femur).

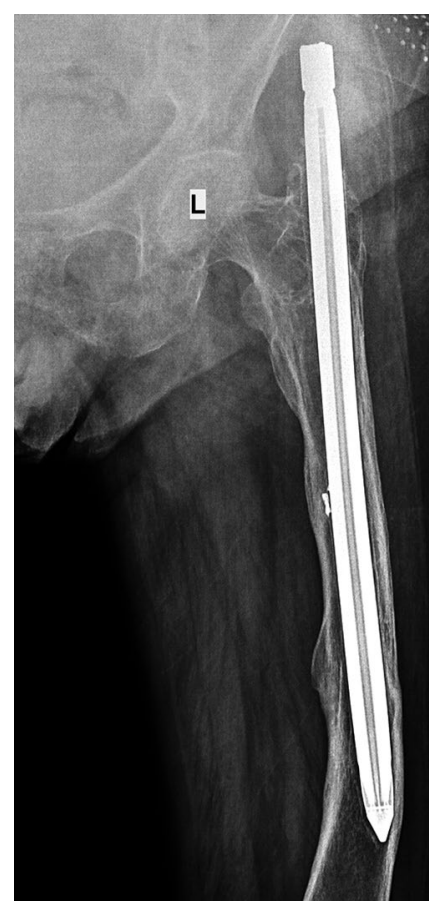

Figure 3. X-ray image of Case 2 (left femur). 
thigh pain, suggesting more severe thigh pain in the pre-fracture period. The patient was also followed for anxiety disorder and treated quetiapine $400 \mathrm{mg} /$ day. Her medical history revealed that alendronate sodium $70 \mathrm{mg}$ was started once a week due to osteoporosis about 10 years ago and she used this treatment regularly for four years. In the third year of alendronate treatment, severe pain began in both thighs and atypical displacement fractures developed. The right femoral shaft was operated six years ago, while the left femoral shaft was operated five and a half years ago and the right subtrochanteric region was operated five years ago without trauma. One year before the displacement fractures, severe thigh pain significantly reduced after fixation surgeries for displacement fractures. Her medical history revealed no history of glucocorticoid use. On physical examination, the range of motion of the hip joint was slightly restricted and painful. The plain radiographs of the patient demonstrated a total hip prosthesis on the right and plate-screws on the femoral shaft, and an intramedullary nail on the left (Figures 2 and 3). Serum PTH, Ca, P, ALP, 25(OH)2D, $\mathrm{Hb}, \mathrm{CBC}, \mathrm{ESR}$, and CRP values were normal. She was informed that she should not take bisphosphonate and about possible risk for falling. No complications were detected in the following visits. A written informed consent was obtained from both patients.

\section{DISCUSSION}

Osteoporosis is an important health problem associated with mortality and morbidity, particularly in the elderly. ${ }^{[2]}$ Bisphosphonates are antiresorptive drugs commonly used in the treatment of osteoporosis. It has been proved in many studies that bisphosphonates increase bone mineral density and reduces fracture risk. ${ }^{[2]}$ However, bisphosphonates are known to have some long- and short-term adverse effects. Long-term bisphosphonate use may result in atypical femoral shaft and subtrochanteric fractures. ${ }^{[1]}$ Bisphosphonate-induced AFF is also responsible for the lack of osteoclastic activity required for the suppression of bisphosphonateinduced bone formation and, consequently, for the repair of damage caused by daily microtraumas. ${ }^{[7]}$ For spontaneous or minimal trauma for AFFs, typical findings on plain radiographs include lateral cortical thickening and transverse fracture line in the femoral shaft and subtrochanteric region. ${ }^{[8]}$

In an AFF case presented by Alshahrani and Kendler, ${ }^{[8]}$ there was a nine-year history of bisphosphonate use. In addition, Wolin et al. ${ }^{[9]}$ reported a case who developed AFF after an eight-year bisphosphonate use. In the literature, there is no case of bisphosphonate-induced AFF in patients using bisphosphonate for less than four years. Our first case had a three-year history of bisphosphonate use. In our second case, bilateral thigh pain started during the third year of alendronate treatment. Although there are cases of bilateral AFF after long-term use of bisphosphonates in the literature, there is no study investigating bilateral multiple fractures in patients using bisphosphonates. Unlike the cases in the literature, our first case had multiple ${ }^{[4]}$ fracture lines. In our second case, AFF developed three times.

Furthermore, the presented cases are expected to contribute to the literature in terms of presenting the first case report on the development of bisphosphonateinduced AFF in members of the same family. In our patients who had normal ALP levels, there were no findings suggestive of hypophosphatasia such as dental problems, abnormal facial appearance, nephrocalcinosis, and craniosynostosis. In addition, there was no findings suggestive of pycnodysostosis characterized by short stature, osteosclerosis, poor pathological fractures, acroosteolysis of terminal phalanges, and craniofacial dysmorphism. Although no signs or symptoms suggestive of osteopetrosis, osteogenesis imperfecta, or similar metabolic bone diseases were detected in our patients, all these should be kept in mind in the presence of AFF in the members of the same family.

Variability in side effects due to bisphosphonate in patients; It may be caused by the interpersonal difference of genes related to osteoclasts and bone cycle affected by bisphosphonates. ${ }^{[6]}$ Although the family history in our case supports this view, many recent studies have illustrated variability in target genes and their relationship with bone mass and other determinants of fracture risk such as bone characteristics, skeletal geometry, and bone turnover markers. The importance of genetic factors in bone quality, treatment response to osteoporosis, and bone turnover has been also proven. ${ }^{[6,10-12]}$ In the presented cases, the emergence of AFF in a shorter time and the development of multiple AFFs can be explained by the genetic predisposition of the patients to AFF. These two cases may shed light into the genetic basis of bisphosphonate-induced AFFs. However, we were unable to perform genetic testing in our patients. Further large-scale, prospective studies using molecular and genetic analyses would provide more accurate results on this topic. 
Although there are some positive results regarding the use of teriparatide in the treatment of AFF, prophylactic stabilization is a more common and valid treatment approach. ${ }^{[13,14]}$ In the first case, similarly, we consulted the orthopedics department for surgery, and the patient was planned to undergo prophylactic intramedullary nailing. However, she refused surgery and, therefore, was discharged with recommendations. There were no complications in her subsequent visits.

In conclusion, family history should be carefully questioned in patients who will undergo osteoporosis treatment and patients taking bisphosphonates should be evaluated for possible AFFs, if they suffer from thigh pain. We believe further studies are needed to examine the relationship between familial predisposition to AFF and bisphosphonate use.

\section{Declaration of conflicting interests}

The authors declared no conflicts of interest with respect to the authorship and/or publication of this article.

\section{Funding}

The authors received no financial support for the research and/or authorship of this article.

\section{REFERENCES}

1. Saita Y, Ishijima M, Kaneko K. Atypical femoral fractures and bisphosphonate use: current evidence and clinical implications. Ther Adv Chronic Dis 2015;6:185-93.

2. Tella SH, Gallagher JC. Prevention and treatment of postmenopausal osteoporosis. J Steroid Biochem Mol Biol 2014;142:155-70.

3. Lovy AJ, Koehler SM, Keswani A, Joseph D, Hasija R, Ghillani R. Atypical femur fracture during bisphosphonate drug holiday: a case series. Osteoporos Int 2015;26:1755-8.

4. Urano T, Inoue $S$. Recent genetic discoveries in osteoporosis, sarcopenia and obesity. Endocr J 2015;62:475-84.
5. Wang $\mathrm{C}$, Zheng $\mathrm{H}$, He JW, Zhang $\mathrm{H}$, Yue $\mathrm{H}, \mathrm{Hu} \mathrm{WW}$, et al. Genetic polymorphisms in the mevalonate pathway affect the therapeutic response to alendronate treatment in postmenopausal Chinese women with low bone mineral density. Pharmacogenomics J 2015;15:158-64.

6. López-Delgado L, Riancho-Zarrabeitia L, Riancho JA. Genetic and acquired factors influencing the effectiveness and toxicity of drug therapy in osteoporosis. Expert Opin Drug Metab Toxicol 2016;12:389-98.

7. Mashiba T, Turner CH, Hirano T, Forwood MR, Johnston CC, Burr DB. Effects of suppressed bone turnover by bisphosphonates on microdamage accumulation and biomechanical properties in clinically relevant skeletal sites in beagles. Bone 2001;28:524-31.

8. Alshahrani F, Kendler D. Femoral fractures in osteoporotic patients on bisphosphonates. A case report. J Clin Densitom 2012;15:380-4.

9. Wolin EA, Banks KP, Vroman PJ. Bisphosphonateassociated atypical subtrochanteric femur fracture. J Nucl Med Technol 2015;43:72-3.

10. Funck-Brentano T, Ostertag A, Debiais F, Fardellone P, Collet C, Mornet E, et al. Identification of a p.Arg708Gln variant in COL1A2 in atypical femoral fractures. Joint Bone Spine 2017;84:715-8.

11. Pérez-Núñez I, Pérez-Castrillón JL, Zarrabeitia MT, García-Ibarbia C, Martínez-Calvo L, Olmos JM, et al. Exon array analysis reveals genetic heterogeneity in atypical femoral fractures. A pilot study. Mol Cell Biochem 2015;409:45-50.

12. Nguyen $H H$, van de Laarschot DM, Verkerk AJMH, Milat F, Zillikens MC, Ebeling PR. Genetic Risk Factors for Atypical Femoral Fractures (AFFs): A Systematic Review. JBMR Plus 2018;2:1-11.

13. Bubbear JS. Atypical Femur Fractures in Patients Treated with Bisphosphonates: Identification, Management, and Prevention. Rambam Maimonides Med J 2016;7:e0032.

14. Oh CW, Oh JK, Park KC, Kim JW, Yoon YC. Prophylactic nailing of incomplete atypical femoral fractures. ScientificWorldJournal 2013;2013:450148. 